\title{
Overexpression of Oct4 in porcine ovarian stem/stromal cells enhances differentiation of oocyte-like cells in vitro and ovarian follicular formation in vivo
}

Yeon-Mi Lee ${ }^{1}$, Tae-Ho Kim', Jeong-Hyeon Lee ${ }^{1}$, Won-Jae Lee ${ }^{1}$, Ryoung-Hoon Jeon', Si-Jung Jang ${ }^{1}$, Sun-A Ock², Sung-Lim Lee ${ }^{1}$, Bong-Wook Park ${ }^{3}$ and Gyu-Jin Rho ${ }^{1,4^{*}}$

\begin{abstract}
Background: Recent findings have revealed that the female gonad may have regenerative activity with having germ line stem cells in juveniles and adults. Application of these germ line stem cells could be an alternative therapy for reproductive disorders in regenerative medicine.

Methods: To enhance the potency of differentiation into oocyte-like cells (OLCs) and folliculogenesis, we overexpressed Oct4 in ovarian stem/stromal cell (OvSCs) and examined the cellular properties related to stemness and self-renewal ability and finally demonstrated the ability of in vitro differentiation and folliculogenesis.

Results: Ovarian cortex included putative stem cells in terms of AP activity, cell cycle status, cell proliferation, expression of mesenchymal lineage surface markers and pluripotent transcriptional markers. Further, Oct4 transfected OvSCs (Oct4-OvSCs) were enhanced their AP activity and cell proliferation compared to OvSCs. The potential on in vitro differentiation into OLCS and in vivo folliculogenesis was also evaluated in OvSCs and Oct4-OvSCs, respectively. Oct4-OvSCs possessed higher oogenesis potential in vitro than OvSCs, in terms of expression of germ cell markers by RT-PCR and the number of OLCs. When OvSCs and Oct4-OvSCs were xeno-transplanted into infertile mice ovaries, the OvSCs transplantation induced new primary follicle formation and hormonal levels of estradiol and FSH remained similar to that of normal mice. However, Oct4-OvSCs possessed higher ability for folliculogenesis based on inducing developing follicles with thecal layer and granulosa cells and more similar estradiol level to normal mice.

Conclusions: These findings demonstrated that putative stem cells were present in ovarian cortex and exhibited differentiation ability into OLCs and folliculogenesis in vivo, and Oct4-overexpression enhanced these ability, suggesting their cellular models based on gene therapy in understanding the mechanisms of oogenesis and folliculogenesis, and finally in view of reproductive cell therapy.
\end{abstract}

Keywords: Oocyte-like cells, Folliculogenesis, Oct4-overexpression, Estradiol, FSH, Porcine

\footnotetext{
* Correspondence: jinrho@gnu.ac.kr

${ }^{1}$ Department of Theriogenology and Biotechnology, College of Veterinary

Medicine, Gyeongsang National University, 501, Jinju-daero, Jinju 660-701,

Republic of Korea

${ }^{4}$ Research Institute of Life Sciences, Gyeongsang National University, 501,

Jinju-daero, Jinju 660-701, Republic of Korea

Full list of author information is available at the end of the article
} 


\section{Background}

The prevailing belief was that all oocytes in mammals originate from the fetal period of life and persist, but decrease in their number through the reproductive phase of females. However, the recent findings have shown that the female gonad of juveniles and adults may have regenerative activity and possess germ line stem cells, which can be considered as an alternative regenerative medicine therapy for reproductive disorders.

Germline stem cells derived from the mouse ovaries gave rise to oocytes and follicles [1, 2], and following ovarian transplantation, the cells completed oogenesis and mating resulted in the birth of offspring [3]. In addition, oocytes-like cells (OLCs) were generated in vitro using stem cells derived from extra-gonadal origins, including bone marrow and peripheral blood [4], skin [5-7], pancreas [8] and adipose tissue [9]. Based on the above findings, multipotent stem cells might possess the ability to generate OLCs under specific culture conditions.

It has been reported that the ovarian stem/stromal cells (OvSCs) are undifferentiated, self-renewal with multi-lineage differentiation capacity into adipocytes, osteocytes, chondrocytes and neurons in vitro [9]. Similar to mesenchymal stem cells (MSCs), which shows ability for self-renewal and differentiation into multiple mesenchymal lineages in vitro [10], OvSCs also known to express Oct4, Sox 2 and Nanog, which are considered to be an important early transcription factors responsible for the regulation of stem cells pluripotency, and revealed the expression of mesenchymal lineage-cell surface markers such as CD44, CD29 and CD90 [11]. However, the evaluation of germ cell specific markers [growth differentiation factor 9b (GDF9b), C-Mos, deleted in azoospermia-like (DAZL), Vasa, zona pellucida C (ZPC), Stella and c-kit] and folliculogenesis marker [follicular stimulating hormone receptor (FSHR)] expression is an important criteria for characterizing OLCs in addition to the assessment of pluripotent transcriptional marker (Oct4) expression [12-14].

In humans, it has been reported that the germ line stem cells exist in ovaries after birth and had the potency for oogenesis in vitro and in vivo. The occurrence of putative ovarian stem cells with no primordial germ cell characteristics which develop into OLCs and blastocyst-like structures was also reported in the ovarian surface epithelium [15]. A recent study has demonstrated that the ovaries of reproductive-aged women possess oogonial stem cells, and these cells were not only being propagated in vitro, but they also generated oocytes in vitro and in vivo [16]. Additionally, a new technology has also been developed to generate transgenic offspring's by transplantation of female germ cells, transfected with exogenous genes, directly into ovaries $[3,17]$. Previously it has been demonstrated that the injection of human germline stem cells isolated from the ovarian cortical biopsies lead to the formation of oocytes after xeno-transplantation into mice [16]. However, additional studies are required in large animal model such as pig, which has both physiological and genetical characteristics similar to humans, rather than relying solely on mice model to understand the mechanism of oogenesis.

Numerous genes are involved in regulating the differentiation ability of stem cells into germ cells [5, 18, 19]. Further, overexpression of Vasa in human embryonic stem cells (ESCs) and induced pluripotent stem cells (iPSCs) has promoted the meiotic progression of germ cells in vitro [20].

Oct4, a regulator of pluripotency [11, 21, 22] expressed in pluripotent stem cells, can induce pluripotency in somatic cells upon transfection [22]. Nevertheless, Oct4 is also expressed in germ cells, which retain the pluripotency through embryogenesis [11]. Recent studies have shown that germline stem cells derived from ovaries also possessed pluripotency as evidenced by the expression of Oct4 [2, 3]. However, no study has been investigated whether the overexpression of Oct 4 affects the differentiation potential of ovarian stem/stromal cells (OvSCs) into female germ cells. Therefore, we hypothesized that the overexpression of Oct4 in undifferentiated OvSCs is liable to increase the efficiency of differentiation into OLCs.

The present study mainly focused on characterizing the cellular properties of OvSCs and Oct4 transfected OvSCs (Oct4-OvSCs) by evaluating the alkaline phosphatase (AP) activity, cell cycle status and cell proliferation as well as expression of cell surface markers and pluripotent transcriptional markers. OvSCs and Oct4-OvSCs differentiated into OLCs were further examined for their ability to initiate and undergo oogenesis in vitro by assessing the morphological characteristics, expression of germ cell specific markers, and measuring the number and size of OLCs. Finally, in vivo folliculogenesis was evaluated by transplanting OvSCs and Oct4-OvSCs into infertile mice.

\section{Methods}

\section{Chemicals and media}

All chemicals and media were purchased from Sigma (St. Louis, MO, USA) and Gibco (Life Technologies, Burlington, ON, Canada), respectively, unless otherwise specified. Media employed for washing was Dulbecco's phosphate buffered saline (D-PBS) supplemented with $1 \mathrm{mg} / \mathrm{ml}$ poly vinyl alcohol (PVA), $1 \%$ penicillinstreptomycin $(10,000 \mathrm{IU}$ and $10,000 \mu \mathrm{g} / \mathrm{ml}$, respectively, Pen-Strep). Advanced Dulbecco's modified Eagle's medium (A-DMEM) supplemented with $10 \%$ fetal bovine serum (FBS), 2 mM glutamine and $1 \%$ Pen-Strep was used for cell 
culture. The $\mathrm{pH}$ and osmolality of all media were adjusted to 7.2 and $285 \pm 5 \mathrm{mOsm} / \mathrm{L}$, respectively.

\section{Isolation and culture of ovarian stem/stromal cells (OvSCs)}

All experiments were authorized by the Animal Center for Biomedical Experimentation at Gyeongsang National University. The two types of cells [i.e., OvSCs and adult fibroblasts (AFs)] were established from sexually mature 6-month-old female pigs. To isolate OvSCs, ovarian cortex was separated using fine forceps and blade under a microscope, minced into small pieces and then digested by incubating in DPBS containing $1 \mathrm{mg} / \mathrm{ml}$ collagenase type I for $1 \mathrm{~h}$. Isolated cells were passed through 100 and $40 \mu \mathrm{m}$ cell strainer (BD Falcon, MA, USA) sequentially to harvest single cell suspension and washed twice with D-PBS by centrifugation at $500 \times \mathrm{g}$ for $5 \mathrm{~min}$. AFs were isolated from ear skin tissues. All the cells were cultured in A-DMEM supplemented with $10 \% \mathrm{FBS}$ at $38.5{ }^{\circ} \mathrm{C}$ in humidified atmosphere of $5 \% \mathrm{CO}_{2}$ in air. Once confluence reached, ovarian stem/stromal cells (OvSCs) were trypsinized using $0.25 \%$ trypsin-ethylenediaminetetraacetic acid (EDTA) solution and pelleted by centrifugation at $500 \times \mathrm{g}$ for $5 \mathrm{~min}$. Cells were replated and passaged 4 times for further experiments.

\section{Transfection of exogenous Oct4 into OvSCs}

Oct4 transfection was followed as per the recommended protocol (Allele biotechnology, CA, USA) with minor modifications. Briefly, a total of $1 \times 10^{5} \mathrm{OvSCs}$ were transfected with $20 \mathrm{MOI}$ hOct3/4-RFP $\left(\mathrm{O}^{\mathrm{R}}\right)$ (ABP-SC-LVIOCT3R, Allele) (Oct4-OvSCs) and polybrene (final concentration, $4 \mu \mathrm{g} / \mathrm{ml}$ ) in $2 \mathrm{ml} \mathrm{A-DMEM}$ supplemented with $10 \% \mathrm{FBS}$ by incubating at $38.5{ }^{\circ} \mathrm{C}$ in a humidified atmosphere of $5 \% \mathrm{CO}_{2}$ in air. Media was changed $10 \mathrm{~h}$ after transfection. OvSCs were also transfected by GFP as a lentiviral infection control. GFP construct was provided by hOct3/4-RFP $\left(\mathrm{O}^{\mathrm{R}}\right)$ kit and the transfection method is same as hOct3/ 4-RFP $\left(\mathrm{O}^{\mathrm{R}}\right)$ transfection.

\section{Alkaline phosphatase (AP) activity}

To detect AP activity, the OvSCs attached to the plastic culture surface were fixed with $3.7 \%$ formaldehyde for $1 \mathrm{~h}$. and washed three times with Tris-HCl buffer [100 mM Tris-HCl (Trizma ${ }^{\odot}$ hydrochloride), $50 \mathrm{mM}$ $\mathrm{NaCl}, 50 \mathrm{mM} \mathrm{MgCl} 2 \cdot 6 \mathrm{H} 2 \mathrm{O}$ and $0.1 \%$ Tween-20]. The cells were stained with AP chromogen kit (BCIP/NBT) (Promega, WI, USA) for $30 \mathrm{~min}$ at room temperature. After being washed twice with deionized water, the cells were evaluated for positive activity under a microscope. AFs were used as a negative control.
Cell cycle, cell proliferation and cell surface markers

The cells at passage 4 with approximately $80 \%$ confluence were analyzed for the stage of cell cycle by fluorescence-activated cell sorting (FACS, Becton Dickinson, NJ, USA). Briefly, after fixation with $70 \%$ ethanol solution at $4{ }^{\circ} \mathrm{C}$ overnight, the cells were washed with D-PBS twice and stained with $10 \mu \mathrm{g} / \mathrm{ml}$ propidium iodide in the presence of $2 \mathrm{mg} / \mathrm{ml}$ RNase A (Qiagen, Hilden, Germany) for $1 \mathrm{~h}$. at $4{ }^{\circ} \mathrm{C}$. A total of $1 \times 10^{4}$ cells were analyzed for the DNA content in triplicates by flow cytometer and the DNA content of each cells was categorized as G0/G1, S or G2/M stage of the cell cycle.

For analysis of cell proliferation, cells were seeded at $1 \times 10^{3}$ cells/well in a 12 -well tissue culture dish in A-DMEM supplemented with $10 \%$ FBS for 14 days. Total cell number of each well was counted with a hematocytometer at every 2 days interval.

Cells were analyzed for the expression of CD markers using flow cytometer. The cells at $\sim 80 \%$ confluence were harvested with $0.25 \%$ trypsin EDTA treatment and blocked with $3 \%$ bovine serum albumin (BSA) solution for $1 \mathrm{~h}$. at $4{ }^{\circ} \mathrm{C}$. For evaluation of CD29 expression, cells were incubated in primary antibody (Mouse anti-pig CD29, $5 \mu \mathrm{g} / \mathrm{ml}, \mathrm{BD}$ Pharmingen ${ }^{\mathrm{TM}}$, CA, USA) for $1 \mathrm{~h}$. at $4{ }^{\circ} \mathrm{C}$. The cells were washed twice using D-PBS and incubated with fluorescein isothiocyanate (FITC)-conjugated secondary antibody (FITC goat anti-mouse IgG, $5 \mu \mathrm{g} / \mathrm{ml}$, BD Pharmingen $^{\mathrm{TM}}$ ) for $1 \mathrm{~h}$. at $4{ }^{\circ} \mathrm{C}$. For analysis of CD44, 45 and 90, cells were incubated with FITC-conjugated antiCD44, $90\left(5 \mu \mathrm{g} / \mathrm{ml}, \mathrm{BD}\right.$ Pharmingen $\left.{ }^{\mathrm{r \mu}}\right)$ and CD45 $(2 \mu \mathrm{g} / \mathrm{ml}$, Sigma, MO, USA) for $1 \mathrm{~h}$. at $4{ }^{\circ} \mathrm{C}$. The standard was established by appropriate isotype-matched negative controls. FITC-labeled cells were analyzed after acquisition of $1 \times 10^{5}$ events by FACS caliber and Cell Quest software (Becton Dickinson). All experiments were performed in triplicates in three independent experiments.

\section{Immunofluorescence staining}

Cells were fixed with $3.7 \%$ formaldehyde solution for $1 \mathrm{~h}$. at room temperature. After being washed twice with $\mathrm{D}$-PBS, the cells were permeabilized with $0.1 \%$ triton X-100 for $30 \mathrm{~min}$ and blocked in D-PBS supplemented with $1 \%$ BSA solution, followed by overnight incubation at $4{ }^{\circ} \mathrm{C}$ with primary antibodies against Oct3/4 (Goat polyclonal, 1:200, Santa Cruz Biotechnology, Inc., CA, USA), Nanog (Goat polyclonal, 1:200, Santa Cruz Biotechnology), Sox2 (Rabbit polyclonal, 1:200, Santa Cruz), VASA (Rabbit polyclonal, 1: 100, Abcam, Cambridge, UK) and DAZL (Rabbit polyclonal, 1:100, Abcam) diluted in blocking solution. Slides were rinsed with D-PBS, and then incubated with FITC-conjugated donkey anti-goat IgG or goat anti-rabbit IgG (1:200, Jackson IR laboratories, Inc., PA, USA) for $45 \mathrm{~min}$ at $38.5{ }^{\circ} \mathrm{C}$. Slides were then counterstaining with $1 \mu \mathrm{g} / \mathrm{ml} 4^{\prime}$,6-diamidino-2-phenylindole 
(DAPI) for $5 \mathrm{~min}$ at room temperature, mounted with Vectashield $^{\bullet}$ (Vector Laboratories, Inc., CA, USA) and observed under a fluorescence microscope (Leica, Wetzlar, Germany).

\section{Reverse transcription-polymerase chain reaction (RT-PCR)}

The expressions of pluripotent transcriptional factors and lineage specific markers were analyzed by RT-PCR. Total RNA was extracted from the cells using RNeasy ${ }^{\circ}$ mini kit (Qiagen, CA, USA) following the manufacturer's protocol. cDNA synthesis was performed for $30 \mathrm{~min}$ at $55{ }^{\circ} \mathrm{C}$ using Omniscript reverse transcription Kit (Qiagen, CA, USA) using oligo-dT primer. PCR was performed using Maxime PCR Premix (iNtRON Biotechnology, Seongnam, Korea) supplemented with each primers and cDNA samples under the following conditions; predenaturation at $95{ }^{\circ} \mathrm{C}$ for $10 \mathrm{~min}$, denaturation at $94{ }^{\circ} \mathrm{C}$ for $30 \mathrm{~s}$, annealing at $56-64{ }^{\circ} \mathrm{C}$ for $30 \mathrm{~s}$, elongation at $72{ }^{\circ} \mathrm{C}$ for $45 \mathrm{~s}$ and final extension at $72{ }^{\circ} \mathrm{C}$ for $10 \mathrm{~min}$ for 30 cycles using a Eppendorf Mastercycler (Eppendorf, Hamburg, Germany). PCR products were loaded on $1 \%$ agarose gel supplemented with $1 \mu \mathrm{g} / \mathrm{ml}$ ethidium bromide solution for electrophoresis. The results were analyzed by zoom browser EX5.7 software (Primetech, Korea). To quantify the gene expression levels, semi-quantitative
RT-PCR was performed, using $\beta$-actin for normalization as a reference gene. The gene expression levels were presented as a fold induction with mean \pm standard error (SE). The intensities of each band were measured by Gel viewer 1.5 (CURVEX Corp., DAIHAN, South Korea) and calculated against a base of $\beta$-actin intensity for relative quantitative analysis. Experiments were performed in triplicates in three independent experiments. The primers used for the study are presented in Table 1. Porcine Oct4 primer (Table 1) was not matched with any sequence in human Oct4 mRNA sequence (GenBank: NM_001285986.1) and it has only matched with porcine Oct4 mRNA Sequence (GenBank: KJ023671.1).

\section{Western blot analysis}

The expression of Oct $3 / 4$, Sox 2 and Nanog proteins were evaluated by western blot analysis. Total protein was extracted with RIPA buffer (PIERCE, IL, USA) containing protease inhibitor, quantified using $\mathrm{BCA}$ protein assay kit (PIERCE, IL, USA). A total of $20 \mu \mathrm{g}$ proteins was separated on SDS-PAGE for $60 \mathrm{~min}$ at $15 \mathrm{~mA}$ per plate and wet transferred electrically onto a nitrocellulose (NC) membrane (Millipore, Eschborn, Germany) for $45 \mathrm{~min}$ at $200 \mathrm{~mA}$. NC membrane was blocked with $5 \%$ skim milk in $0.1 \%$ TBST ( $1 \mathrm{M}$ Tris [pH 7.5], $5 \mathrm{~N}$

Table 1 RT-PCR primers specific to porcine MSCs and differentiated cells

\begin{tabular}{|c|c|c|c|c|}
\hline Gene & Primer sequence $\left(5^{\prime}-3^{\prime}\right)$ & Product Size (bp) & Annealing $\operatorname{Tm}\left({ }^{\circ} \mathrm{C}\right)$ & Reference/Accession number \\
\hline \multirow[t]{2}{*}{ OCT4 } & F-AGGTGTTCAGCCAAACGACC & 335 & 60 & Carlin et al. [12] \\
\hline & R-TGATCGTTTGCCCTTCTGGC & & & \\
\hline \multirow[t]{2}{*}{ SOX2 } & F-GCCTGGGCGCCGAGTGGA & 443 & 64 & Carlin et al. [12] \\
\hline & R-GGGCGAGCCGTTCATGTAGGTCTG & & & \\
\hline \multirow[t]{2}{*}{ Nanog } & F-ATCCAGCTTGTCCCCAAAG & 438 & 60 & Carlin et al. [12] \\
\hline & R-ATTTCATTCGCTGGTTCTGG & & & \\
\hline \multirow[t]{2}{*}{ GDF9b } & F-GGATCCAGAAAAGCACAACC & 227 & 56 & Dyce et al. [5] \\
\hline & R-AGTGTCCAGGGATGAAATGC & & & \\
\hline \multirow[t]{2}{*}{ FSHR } & F-CTCACCTACCCCAGCCACT & 243 & 56 & Dyce et al. [5] \\
\hline & R-CTCAGGGGAGCAAGTCACAT & & & \\
\hline \multirow[t]{2}{*}{ C-MOS } & F-AAATCAGCGACTITGGTTGC & 200 & 56 & Dyce et al. [5] \\
\hline & R-CTGACGCTCCCCTGAGTAAG & & & \\
\hline \multirow[t]{2}{*}{ VASA } & F-TTGCAGGACGAGATTTGATG & 165 & 56 & Dyce et al. [5] \\
\hline & R-CCAATTCTCGAGTTGGTGC & & & \\
\hline \multirow[t]{2}{*}{ ZPC } & F-TGGTGTACAGCACCTTCCTG & 202 & 56 & Dyce et al. [5] \\
\hline & R-ATCAGGCGCAGAGAGAACAC & & & \\
\hline \multirow[t]{2}{*}{ Stella } & F-TTAATCCAACCCGGACTCAG & 173 & 56 & Linher et al. [7] \\
\hline & R-TGGTTGAGGTGGATATTCTGG & & & \\
\hline \multirow[t]{2}{*}{ C-kit } & F-TGTATTCACAGAGACTTGGCGG & 124 & 56 & Linher et al. [7] \\
\hline & R-CGTTCCTTTGACCACGTAA & & & \\
\hline \multirow[t]{2}{*}{ B-Actin } & F-CTCGATCATGAAGTGCGACG & 114 & 58 & SSU07786 \\
\hline & R-GTGATCTCCTTCTGCATCCTGTC & & & \\
\hline
\end{tabular}


$\mathrm{NaCl}$ and $0.1 \%$ Tween-20) for $1 \mathrm{~h}$. at room temperature. After washing 3 times with $0.1 \%$ TBST, the NC membrane was incubated with 1:200 dilution of Oct3/4, Sox2 and Nanog (Santa Cruz Biotechnology, CA, USA) and 1:1000 dilution of $\beta$-actin (Cell Signaling Technology, MA, USA) antibody in $0.1 \%$ TBST - $5 \%$ skim milk for overnight at $4{ }^{\circ} \mathrm{C}$. After being washed 3 times with $0.1 \% \mathrm{TBST}$, the $\mathrm{NC}$ membrane was incubated with 1:5,000 dilution of goat conjugate anti-rabbit IgG-HRP or donkey conjugate anti-goat IgG-HRP (Santa Cruz Biotechnology, CA, USA) in $0.1 \%$ TBST - 5 \% skim milk for $1 \mathrm{~h}$. at room temperature. Immunoreactivity was detected by enhanced chemiluminescence (ECL; Supersignal ${ }^{\odot}$ West Pico Chemiluminescent substrate, PIERCE), and then exposed to X-ray film (FUJI Photo Film Co., Ltd, Tokyo, Japan). To quantify the protein expression levels, semi-quantification was performed. The protein expression levels were normalized to $\beta$-actin protein levels and presented as a fold induction with mean \pm S.E. Experiments were performed in triplicates in three independent experiments. Relative intensities of each band were calculated on the basis of $\beta$-actin intensity by Gel viewer 1.5 (CURVEX Corp., DAIHAN, South Korea).

\section{Differentiation into oocyte-like cells (OLCs) and measuring the number of OLCs}

OvSCs and Oct4-OvSCs were differentiated into OLCs following a previously published protocol [5] with minor modifications. Media for induction into OLCs consisted of DMEM and nutrient mixture F12 (DMEM:F12 = 1:1, v/v) supplemented with $5 \%$ FBS, $5 \%$ porcine follicular fluid, $0.23 \mathrm{mM}$ sodium pyruvate, $0.1 \mathrm{mM}$ non-essential amino acids, $2 \mathrm{mM}$ L-glutamine and $0.1 \mathrm{mM} \beta$ mercaptoethanol. The cells were cultured in differentiation media for 45 days by changing the media twice a week.

Induced OLCs were confirmed by observing their morphology with increasing size to approximately $50 \mu \mathrm{m}$ in diameter at day 15, 30 and 45 of differentiation. On day 45 , OLCs were stained with $1 \mu \mathrm{g} / \mathrm{ml}$ DAPI and observed under a fluorescence microscope (Nikon, Tokyo, Japan). The expression of pluripotent transcriptional factor, Oct4, and germ cell specific markers such as growth differentiation factor 9b (GDF9b), C-Mos, Vasa, ZPC, Stella, c-kit and folliculogenesis marker, FSHR was analyzed by using RT-PCR. $\beta$-actin was used as a control gene. Further, the expressions of Oct3/4, Vasa and DAZL in OLCs were analyzed by immunocytochemistry. In vitro produced porcine mature oocytes were used for positive control for immunocytochemistry.

For evaluating the number of OLCs, cells were seeded at $1 \times 10^{5}$ cells/well in a 24 -well plate and differentiated into OLCs for 45 days. On day 45 , the floating cells in each well were counted and measured the diameter of the cells using ocular micrometer (Nikon TE300, Japan). The OLCs were classified on their diameter into $<50 \mu \mathrm{m}$ and $>50 \mu \mathrm{m}$ in diameter, and if OLCs had zona pellucida, the measurements included its diameter. Experiments were performed in eight replicates in three independent experiments.

\section{Animal preparation and cell transplantation}

Before the cell transplantation, the cells were labeled with fluorescent lipophilic carbocyanine dye PKH26 according to the manufacturer's instructions (Sigma, MO, USA). The labeled cells were then used for transplantation experiments.

Female BALB/C Nude mice (normal mice), aged 5-6 weeks, weighing approximately 18-20 g, were used in this study. To induce infertility, recipients were sterilized by intraperitoneal injection of busulphan $(20 \mathrm{mg} / \mathrm{kg}$, suspended in DMSO), followed by a booster injection after 2 weeks. After 2 weeks from the booster injection, the animals were divided into five groups: control $(\mathrm{n}=5$, not injected with PBS or cells), PBS injection $(n=5)$, AFs injection $(n=7)$, OvSCs injection $(n=10)$, and Oct4OvSCs injection $(n=10)$. After being anesthetized with a combination of $1 \mu \mathrm{l} / \mathrm{g}(60 \mu \mathrm{g} / \mathrm{g})$ Zolazepam/tiletamine (zoletil50, Verbac, France) and $0.5 \mu \mathrm{l} / \mathrm{g}$ Zylazine, mice were injected with $5 \mu \mathrm{l}$ PBS alone or with $5 \mu \mathrm{l}\left(1 \times 10^{4}\right.$ cells) of cell suspensions into ovarian cortex using glass pipettes with a $70 \mu \mathrm{m}$ diameter. Injections of PBS or AFs were evaluated for negative controls in normal and infertile mice.

\section{Histological assessment and hormone measurement}

Sera collected from the mice at 4 weeks after PBS or cell injection was used to measure the estradiol $17 \beta$ and FSH level using ELISA. Estradiol $17 \beta$ and FSH were analyzed using enzyme immunoassay kit for estradiol (Oxford Biomedical Research, MI, USA) and FSH (Endocrine Technology, CA, USA) according to the manufacture's protocol, respectively. Five mice were used for each group and all serum samples and standards were run in duplicate.

The mice were sacrificed at 4 weeks after PBS or cell injection, and ovaries were collected for histological assessment. The ovaries were fixed with $4 \%$ paraformaldehyde for overnight. After being washed with D-PBS 3 times each for $5 \mathrm{~min}$, the ovaries were dehydrated overnight with $20 \%$ sucrose. The dehydrated ovaries were embedded in optical cutting temperature (OCT) compounds (Tissue-Tek ${ }^{\oplus}, \mathrm{CE}, \mathrm{USA}$ ) and sectioned into $8 \mu \mathrm{m}$ thickness and mounted onto slides. The slides were stained with hematoxylin and eosin (H\&E) staining or $1 \mu \mathrm{g} / \mathrm{ml}$ DAPI for $5 \mathrm{~min}$. Images were observed using optical microscope 
(Nikon TE300, Japan) or fluorescence microscope (Leica CTR600, Switzerland).

For immunohistochemistry, the rabbit specific horseradish peroxidase-diaminobenzidine (HRP-DAB) detection immunohistochemical kit (Abcam) was used. Briefly, sections were incubated with a hydrogen peroxide block solution for $10 \mathrm{~min}$, followed by treating protein block solution for $30 \mathrm{~min}$. After being washed by D-PBS, sections were incubated with the primary antibody, anti-estrogen receptor alpha (rabbit polyclonal, $1: 100$, Abcam) at $4{ }^{\circ} \mathrm{C}$ overnight. Biotinylated goat anti rabbit $\operatorname{IgG}(\mathrm{H}+\mathrm{L})$ was treated to section for $10 \mathrm{~min}$ as a secondary antibody. Visualization was detected using HRP-DAB detection IHC kit according to the manufacturer's instructions. After counterstaining with hematoxylin, the sections were mounted and observed under the microscope.

\section{Statistical analysis}

Differences among proportional data were analyzed by SPSS 21.0 (SPSS Inc. Chicago, IL. USA). All data was expressed as means \pm SE. Comparisons of mean values among groups were performed using student $\mathrm{T}$ - test or ANOVA with Tukey's or Duncan's multiple comparisons test. Differences were considered to be significant at $P<0.05$.

\section{Results}

\section{Increased AP activity in Oct4-OvSCs}

In primary culture, the OvSCs were grown as heterogeneous populations with elongated, sphere-like or spindlelike adherent features. However, from passage 2-3, the cells became a homogeneous population with a typical fibroblast-like morphology (Fig. 1Aa). After Oct4 transfection of OvSCs, Oct4-OvSCs were also grown into homogeneous populations with a typical fibroblast-like morphology at passage 4 (Fig. 1Ad). OvSCs were transfected with lentiviral vector which expressed GFP as an infection control, and the infection rate in Oct4-OvSCs was found to be $66.7 \pm 0.7 \%$, while non-transfected cells were not detected for GFP (Fig. 1e). At passage 4, OvSCs

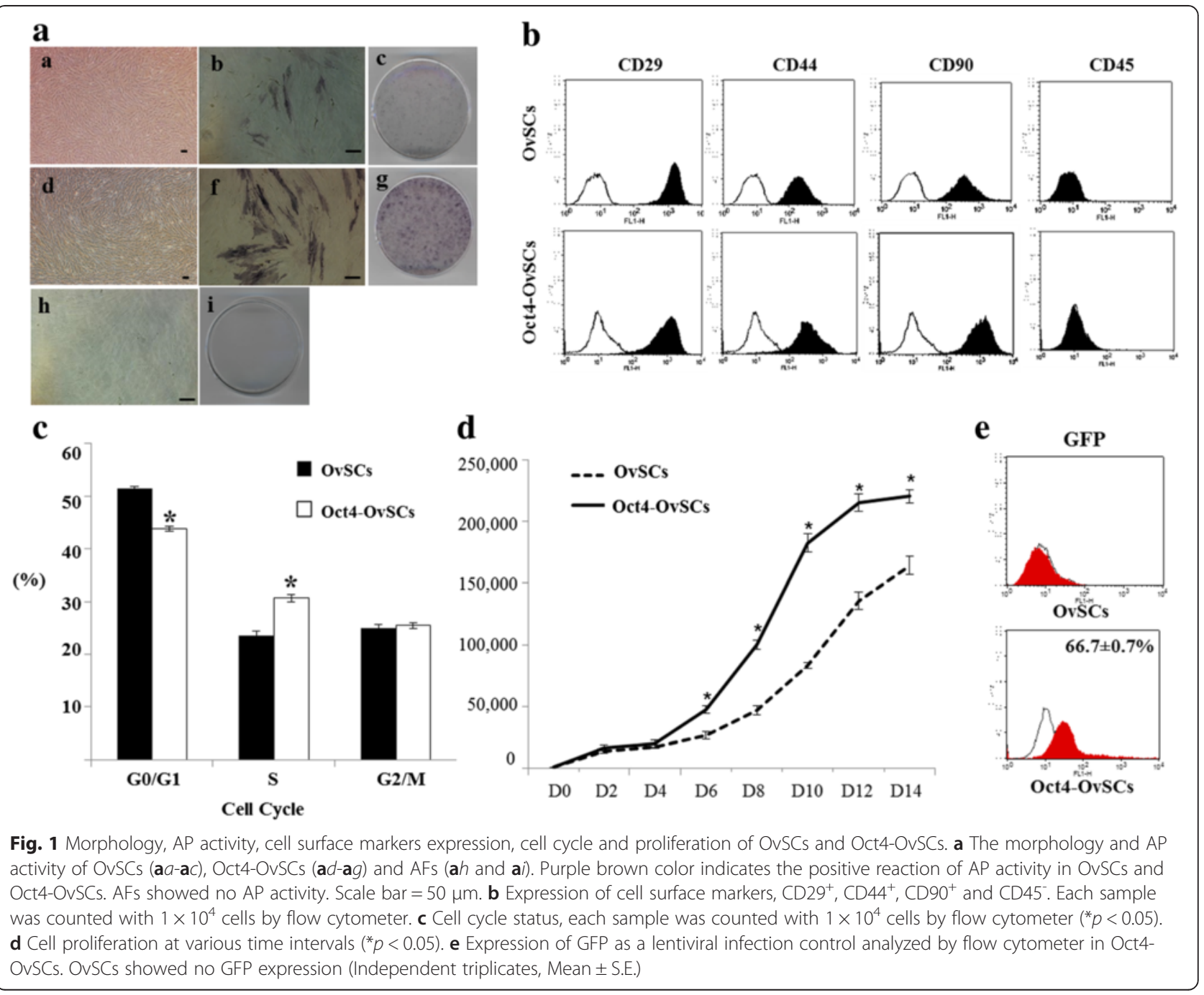


(Fig. $1 \mathrm{Ab}$ and $\mathrm{Ac}$ ) and Oct-OvSCs (Fig. 1Af and Ag) were positive for AP activity and more population were observed for AP positivity in Oct4-OvSCs compared to OvSCs. These results indicated the existence of stem cells in ovarian cortex and proved the potential stemness in OvSCs transfected with exogenous Oct4. AFs were observed to be negative for AP activity (Fig. 1Ah and Ai).

\section{Expression of mesenchymal cell surface markers in OvSCs and Oct4-OvSCs}

The expression of mesenchymal cell surface specific markers, such as CD29, CD44 and CD90 were analyzed in OvSCs and Oct4-OvSCs using flow cytometer at passage 4 (Fig. 1b). The results were presented as percentage mean positive cells of surface markers in analyzed cell population. The percentage of cells that expressed CD44 was significantly $(P<0.05)$ greater in OvSCs than that in Oct4-OvSCs $(98.7 \pm 0.7 \%$ vs. $94.7 \pm 0.3 \%)$. CD29 and CD90 were highly expressed in both OvSCs and Oct4-OvSCs with no significant differences. However, the hematopoietic cell lineage marker, CD45, was not detected in OvSCs and Oct4-OvSCs (Fig. 1b).

\section{Enhanced cell proliferation in Oct4-OvSCs}

Flow cytometer analysis showed that the population of G0/G1 phase of the cell cycle in Oct4-OvSCs was significantly $(P<0.05)$ lower than OvSCs $(43.8 \pm 0.5 \%$ vs. $51.5 \pm 0.3 \%$ ) (Fig. 1c). In contrast, the population of $S$ phase of the cell cycle in Oct4-OvSCs was significantly $(P<0.05)$ higher than OvSCs $(30.7 \pm 0.7 \%$ vs. $23.6 \pm$ $0.8 \%)$. These results indicated that the Oct 4 transfection resulted in enhanced cell proliferation. The population of cells at G2/M phase in Oct4 transfected cells and OvSCs was observed to be $25.5 \pm 0.6$ and $24.9 \pm 0.7$ respectively.

Further, to confirm the cell proliferation ability, OvSCs and Oct4-OvSCs were compared for growth kinetics at passage 4 (Fig. 1d). Growth pattern of these cells revealed a sigmoid curve, wherein proliferation of Oct4OvSCs was significantly $(P<0.05)$ more rapid than OvSCs from day 6 to 14. There was no significant difference in cell proliferation between OvSCs and Oct4OvSCs at day 2 and 4 of culture.

\section{Variation of expression of pluripotency-related transcription factors after Oct4 transfection}

The expression of pluripotent transcriptional factors, such as Oct3/4, Sox 2 and Nanog, in OvSCs and Oct4OvSCs was analyzed by RT-PCR and western blot analysis (Fig. 2). The exogenous Oct4, which was transfected using human Oct3/4 lentiviral particle, was expressed in Oct4-OvSCs, whereas in OvSCs, it was not expressed (Fig. 2a). The endogenous Oct4 was expressed in OvSCs and Oct4-OvSCs as revealed by RT-PCR (Fig. 2a). However, the intensity of endogenous Oct4 was significantly $(P<0.05)$ higher in Oct4-OvSCs than OvSCs (Fig. 2c). The Sox 2 was also expressed in OvSCs and Oct4-OvSCs, and there was no significant difference between these cells based on band intensities (Fig. 2a and c). Nanog was also expressed in OvSCs and Oct4-OvSCs, with significantly $(P<0.05)$ higher level in OvSCs than Oct4OvSCs (Fig. 2a and c). Protein levels of Oct3/4, Sox 2 and Nanog were evaluated by western blot analysis. Oct3/4 protein was expressed only in Oct4-OvSCs, but not in OvSCs (Fig. 2b and d). Sox2 and Nanog were detected in OvSCs and Oct4-OvSCs, with no significant difference among these cells (Fig. $2 b$ and d).

\section{Enhanced OLCs formation and expression of transcriptional and germ cell markers in Oct4-OvSCs}

Before induction into OLCs, OvSCs and Oct4-OvSCs had a fibroblast-like morphology (Fig. 1Aa and Ac). Following the differentiation, the cells changed to a distinct spherelike morphology during the next 15 to 30 days of culture in the differentiation media (OvSCs, Fig. 3Aa and Ab; Oct4-OvSCs, Fig. 3Ba and Bb). At day- 45 of differentiation, the morphology of cells was changed to 'big roundcells', which had approximately $50 \mu \mathrm{m}$ in diameter, so called oocyte-like cells (OLCs) (Fig. 3Ac and Bc), which characteristically possessed zona pellucida like structure. Further, nuclear staining by DAPI was observed in OLCs derived from OvSCs (Fig. 3Ad and Ae) and Oct4-OvSCs (Fig. 3Bd and Ae) under a fluorescent microscope. OLCs derived from Oct4-OvSCs expressed a cytoplasmic red fluorescence protein (Fig. 3Be).

The number and diameter of OLCs derived from Oct4-OvSCs and OvSCs was analyzed by counting the floating cells (Fig. 3c). At day-45 of differentiation, the floating cells were counted and diameter of the cells was measured. The number of floating cells, less than $50 \mu \mathrm{m}$ in diameter, was similar between Oct4-OvSCs and OvSCs $(800.0 \pm 44.3$ vs. $818.7 \pm 38.9)$. However, the OLCs from Oct4-OvSCs were generated significantly $(P<0.05)$ higher number of cells with $>50 \mu \mathrm{m}$ in diameter than OvSCs $(68.7 \pm 1.7$ vs. $51.4 \pm 2.9)$.

To characterize the differentiated cells into OLCs from OvSCs and Oct4-OvSCs, the expression of transcription factor (Oct4), germ cell and meiosis specific markers (Vasa, GDF9b, C-mos, C-kit, Stella and ZPC), and folliculogenesis marker (FSHR) was evaluated at mRNA level using RT-PCR. The relative expression levels were quantified using band intensities (Fig. 4a and b). Ovarian tissues were used as positive control and they expressed all oocyte specific markers (Fig. 4a). Oct4, Vasa, GDF9b, $C$-mos, FSHR and C-kit were detected in OvSCs and Oct4-OvSCs at all time points during the differentiation that were examined (Fig. 4a). Overexpression of 


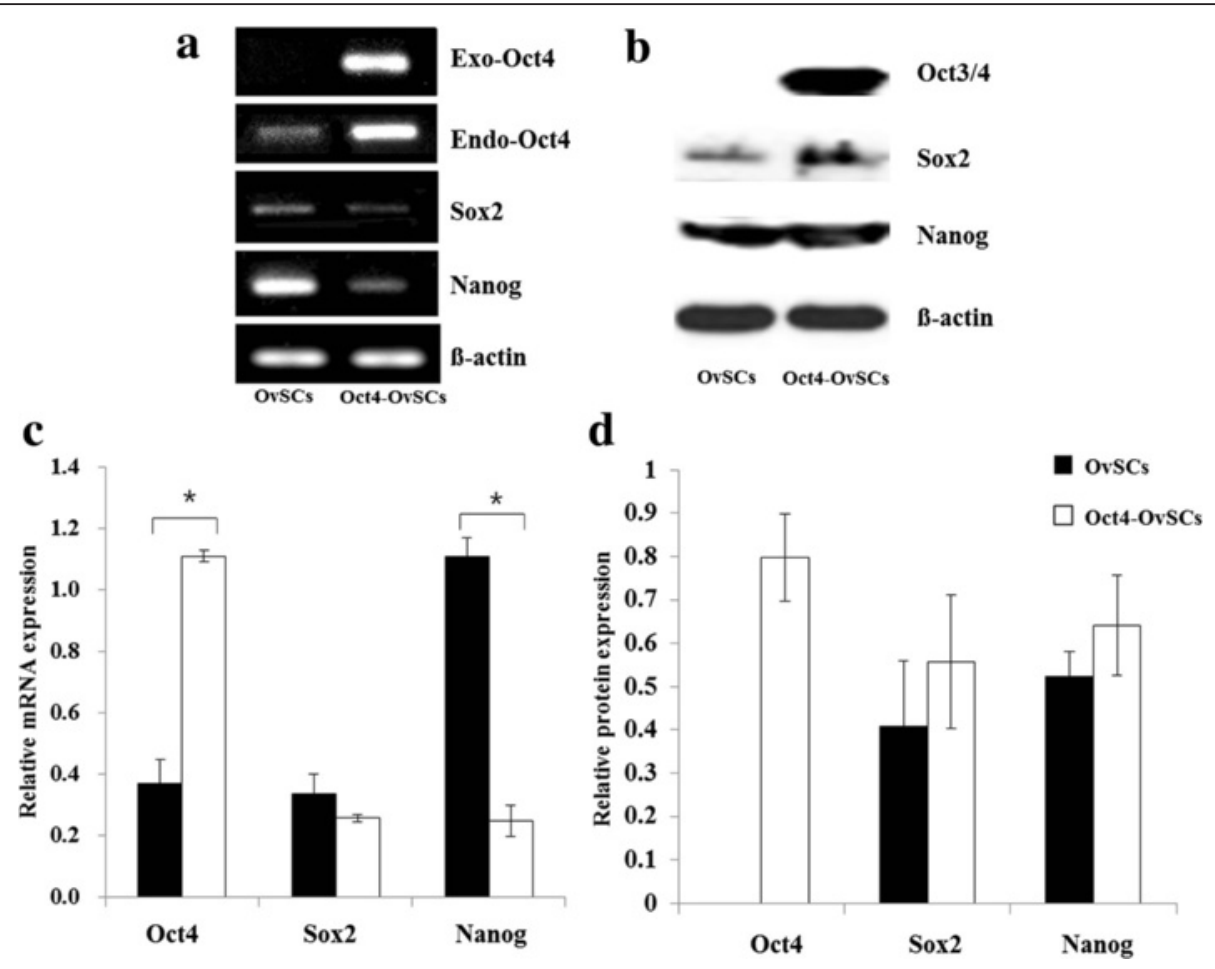

Fig. 2 Expression of pluripotency-related transcription factors in OvSCs and Oct4-OvSCs analyzed by RT-PCR and western blot. a Result of RT-PCR for exogenous Oct4, endogenous Oct4, Sox2 and Nanog. b Western blot analysis for Oct3/4, Sox2 and Nanog. $\beta$-actin was used as a reference. c Relative mRNA abundance of endogenous Oct4, Sox2 and Nanog with a base of b-actin intensity. $\mathbf{d}$ Relative protein abundance of Oct3/4, Sox 2 and Nanog with a base of $\beta$-actin intensity. Three independent experiments performed in triplicates were used for $C$ and $D\left({ }^{*} p<0.05\right)$

Oct4 was displayed with higher level of Oct4 at each point till day 45 of differentiation of Oct4-OvSCs than OvSCs based on band intensities. However, the expression level of Oct4 did not differ between day 0 and 45 of differentiation in both OvSCs and Oct4OvSCs (Fig. 4b). The expression of Vasa was significantly $(P<0.05)$ increased in Oct4-OvSCs after differentiation into OLCs, but not in OvSCs (Fig. 4b). Further, there was no significant difference in the expression of Vasa among OvSCs and Oct4-OvSCs on day 45 of differentiation. GDF9b, C-mos and FSHR were expressed significantly $(P<0.05)$ higher in both OvSCs and Oct4-OvSCs on day 45 of differentiation (Fig. 4b), although differences between cell types were not significant. C-kit level was significantly $(P<0.05)$ higher in Oct4-OvSCs than OvSCs on day 45 of differentiation, but there was no significant difference between day 0 and 45 of differentiation (Fig. 4b). The expression of Stella initiated on day 45 and 15 of differentiation in OvSCs and Oct4OvSCs, respectively (Fig. 4a). However, there was no significant difference in relative mRNA level of Stella between OvSCs and Oct4-OvSCs on day 45 of differentiation (Fig. 4b). The expression of $Z P C$ initiated on day 15 of differentiation in both OvSCs and Oct4-OvSCs, and its expression level was found to be significantly $(P<0.05)$ higher in Oct4 OvSCs compared to OvSCs on day 45 of differentiation (Fig. 4a and b). OLCs derived from OvSCs and Oct4-OvSCs were further analyzed by immunocytochemistry to detect the expression of Oct3/4, Vasa and DAZL at protein level (Fig. 5). Oct3/4 was expressed in the nucleus of OLCs, whereas Vasa and DAZL were detected in the cytoplasm (Fig. 5a and b) and these expression patterns were similar to in vitro matured oocytes (Fig. 5c).

\section{Follicle formation in OvSCs and Oct4-OvSCs transplanted mice}

Control BALB/C nude mice ovary revealed intact follicles containing oocytes and were found to be progressing through folliculogenesis (Fig. 6Aa and Ba). Upon injecting busulphan twice at 2 week intervals resulted in a disruption of folliculogenesis and destruction of germ cells including oocytes in ovaries when observed at 2 and 6 weeks after the second injection (Fig. 6Ab and Ac). A total of $1 \times 10^{4}$ cells were transplanted into ovaries of busulphan treated mice. At 4 weeks after OvSCs transplantation into sterile ovaries, primary follicles were observed in the ovaries (Fig. 6Af and Ag). Furthermore, the ovaries transplanted with Oct4-OvSCs showed not only primary follicles (Fig. 6Ai) but also developing follicles containing theca and granulosa cells (Fig. 6Ah and $\mathrm{Ai}$ ). Under the fluorescence microscopy, Oct4-OvSCs labeled with 


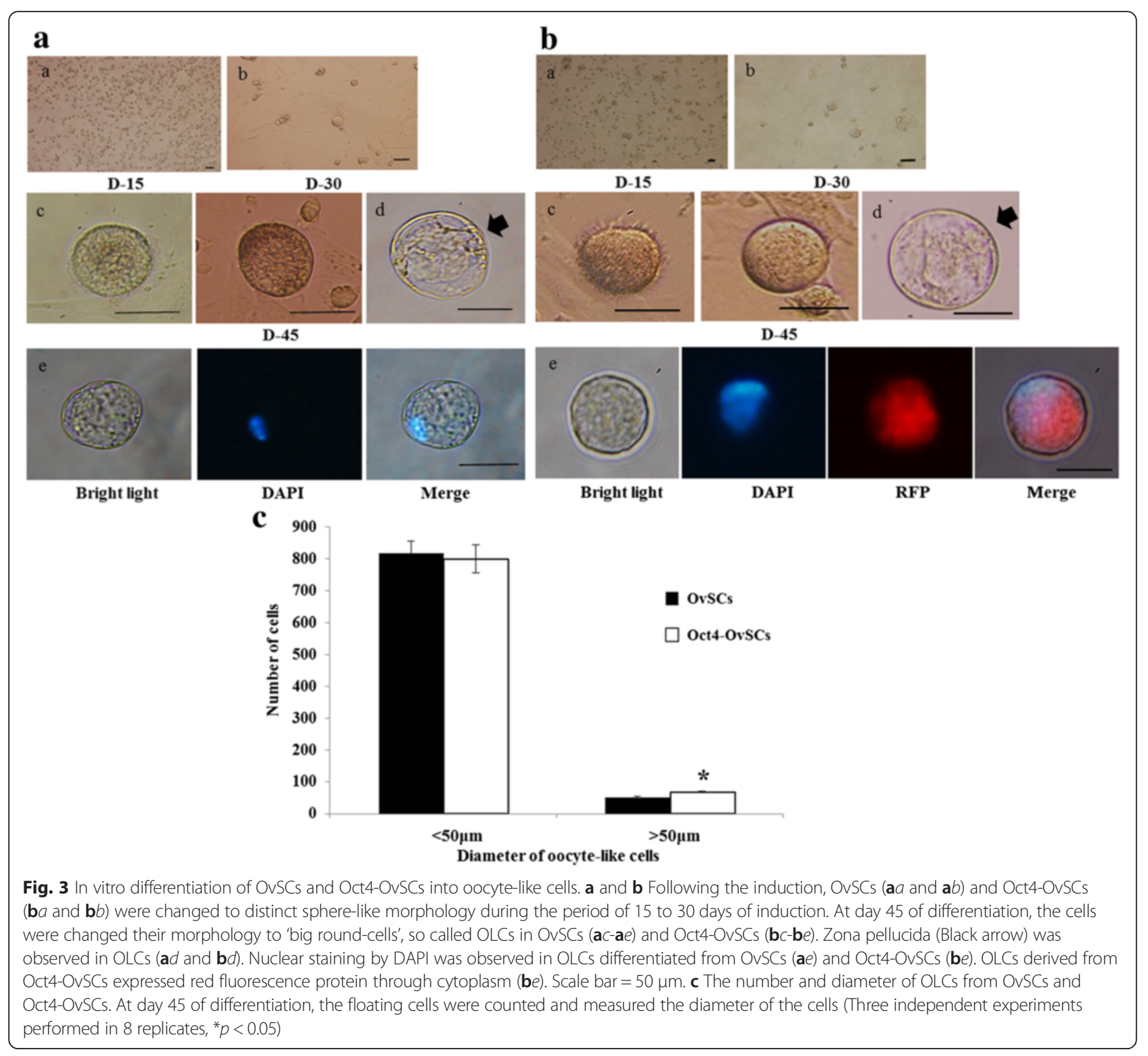

PKH26 were observed in developing follicles (Fig. 6Bf and $\mathrm{Bg}$ ). However, OvSCs and Oct4-OvSCs labeled with PKH26 were not located in primary follicles (Fig. 6Be and $\mathrm{Bh}$ ). The labeled AFs did not induce any follicles in sterile ovaries (Fig. 6Ad and Bc). The labeled cells were not observed in PBS injected ovaries (Fig. 6Ae and Bd).

Estrogen receptor alpha $(E R \alpha)$ was expressed in theca cells and interstitial cells in normal ovaries that were not injected with busulphan (Fig. 7a). After injection of busulphan in mouse, ER $\alpha$ was expressed in all interstitial cells in sterile ovaries (Fig. 7b). AFs or PBS injected ovaries also expressed $E R \alpha$ in all interstitial cells in infertile mice (Fig. 7c and d). In OvSCs transplanted ovaries, ER $\alpha$ was not expressed in primary follicles containing granulosa cells, but expressed in interstitial cells (Fig. 7e). ER $\alpha$ was expressed in theca cells of developing follicles and interstitial cells, but not in primary follicles in Oct4OvSCs transplanted ovaries (Fig. $7 \mathrm{f}$ and $\mathrm{g}$ ).

\section{Normal estradiol and FSH level in OvSCs and Oct4-OvSCs transplanted mice}

To evaluate the concentration of estradiol and FSH in mouse serum, ELISA analysis was performed (Fig. 8a and $\mathrm{b}$, respectively). Control infertile mice revealed significantly $(P<0.05)$ lower estradiol concentration than normal female BALB/C Nude mice $(26.0 \pm 3.3$ vs. $52.7 \pm$ $6.1 \mathrm{pg} / \mathrm{ml}$ ), and showed similar estradiol concentration to AFs or PBS injected infertile mice $(28.5 \pm 3.6$ and $25.75 \pm 5.4 \mathrm{pg} / \mathrm{ml}$ ). However, OvSCs or Oct4-OvSCs transplantation resulted in higher estradiol concentration in infertile mice. The concentrations of estradiol in OvSCs injected mice $(45.5 \pm 3.3 \mathrm{pg} / \mathrm{ml})$ were lower 


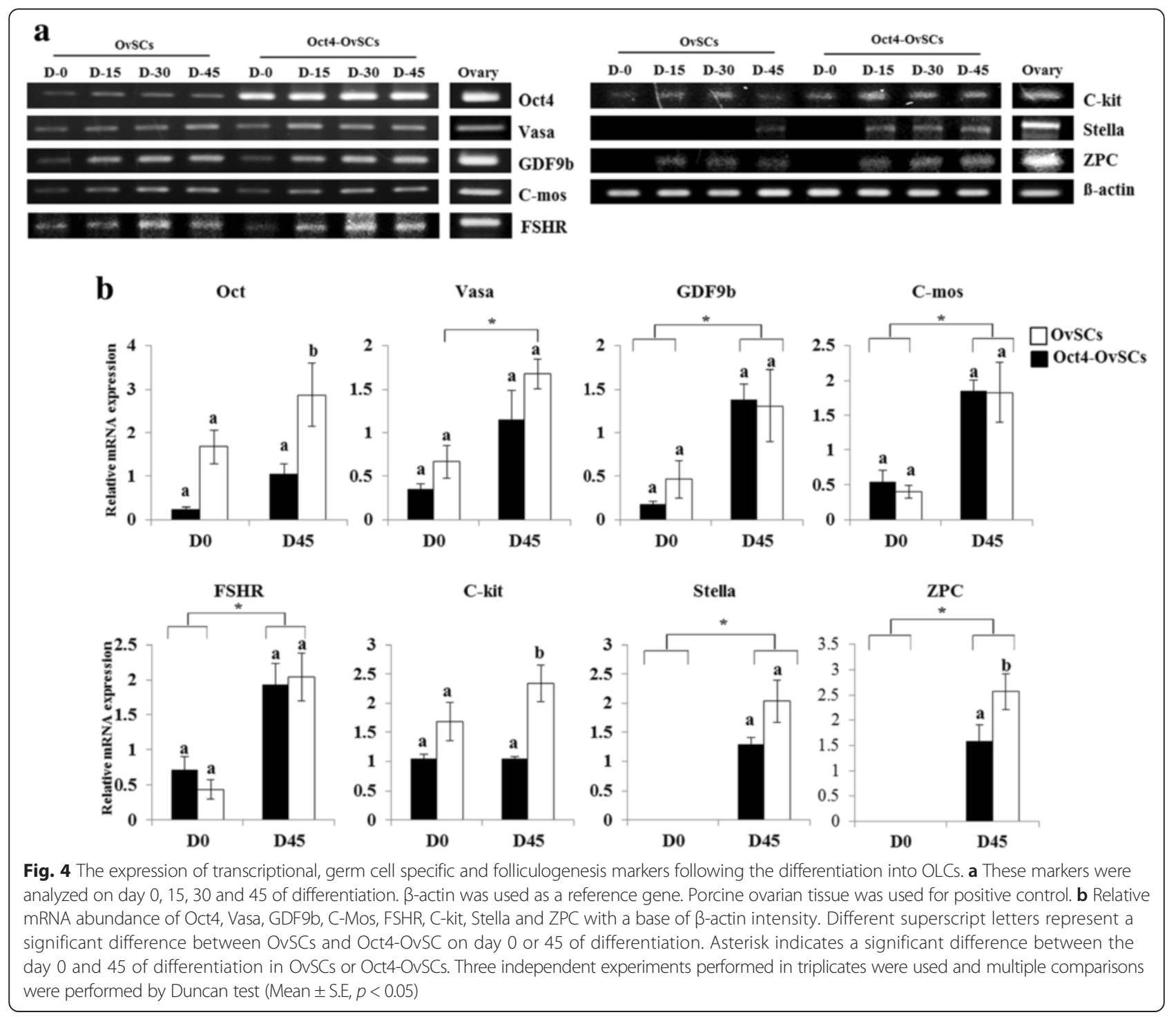

than normal and Oct4-OvSCs injected mice (56.5 \pm $4.3 \mathrm{pg} / \mathrm{ml}$ ), but there was no significant difference among these groups.

FSH concentration of control infertile mice was significantly $(P<0.05)$ higher than normal mice $(62.5 \pm 13.2$ vs. $5.1 \pm 0.9 \mathrm{ng} / \mathrm{ml}$ ). After injection of AFs or PBS into infertile mice, FSH concentration was still significantly $(P<0.05)$ higher than normal mice $(59.1 \pm 12.0$ and $61.6 \pm 16.3$ vs. $5.1 \pm 0.9 \mathrm{ng} / \mathrm{ml})$. However, FSH concentration decreased by transplantation of OvSCs or Oct4OvSCs into infertile mice. OvSCs or Oct4-OvSCs injected mice had lower FSH concentration than control infertile mice $(23.8 \pm 5.4$ and $21.8 \pm 4.6$ vs. $62.5 \pm 13.2 \mathrm{ng} / \mathrm{ml})$. The FSH concentration of OvSCs or Oct4-OvSCs was higher than normal mice, but there was no significant difference.

\section{Discussion}

Ovarian stem cells, commonly isolated from ovarian surface epithelium or ovarian germinal epithelium [15], are also found in ovarian cortex enclosed by epithelial like cells of irregular shape. Recent study has reported two different types of stem cells in the ovary named as ovarian germ stem cells (OGSCs) and very small embryoniclike stem cells (VSELs) [23]. OvSCs used in this study showed fibroblast like morphology with a size of $10-$ $15 \mu \mathrm{m}$. Therefore, OvSCs in the present study are comparable with OGSCs $($ size $>8 \mu \mathrm{m})$ than VSEL $(3-5 \mu \mathrm{m})$. Further, VSEL expresses nuclear OCT4, while OGSE expresses cytoplasmic OCT4 at protein level, suggesting VSELs are more pluripotent. In the present study OvSCs did not express Oct3/4 protein suggesting their less pluripotent status. Therefore, in our view, OvSCs used 


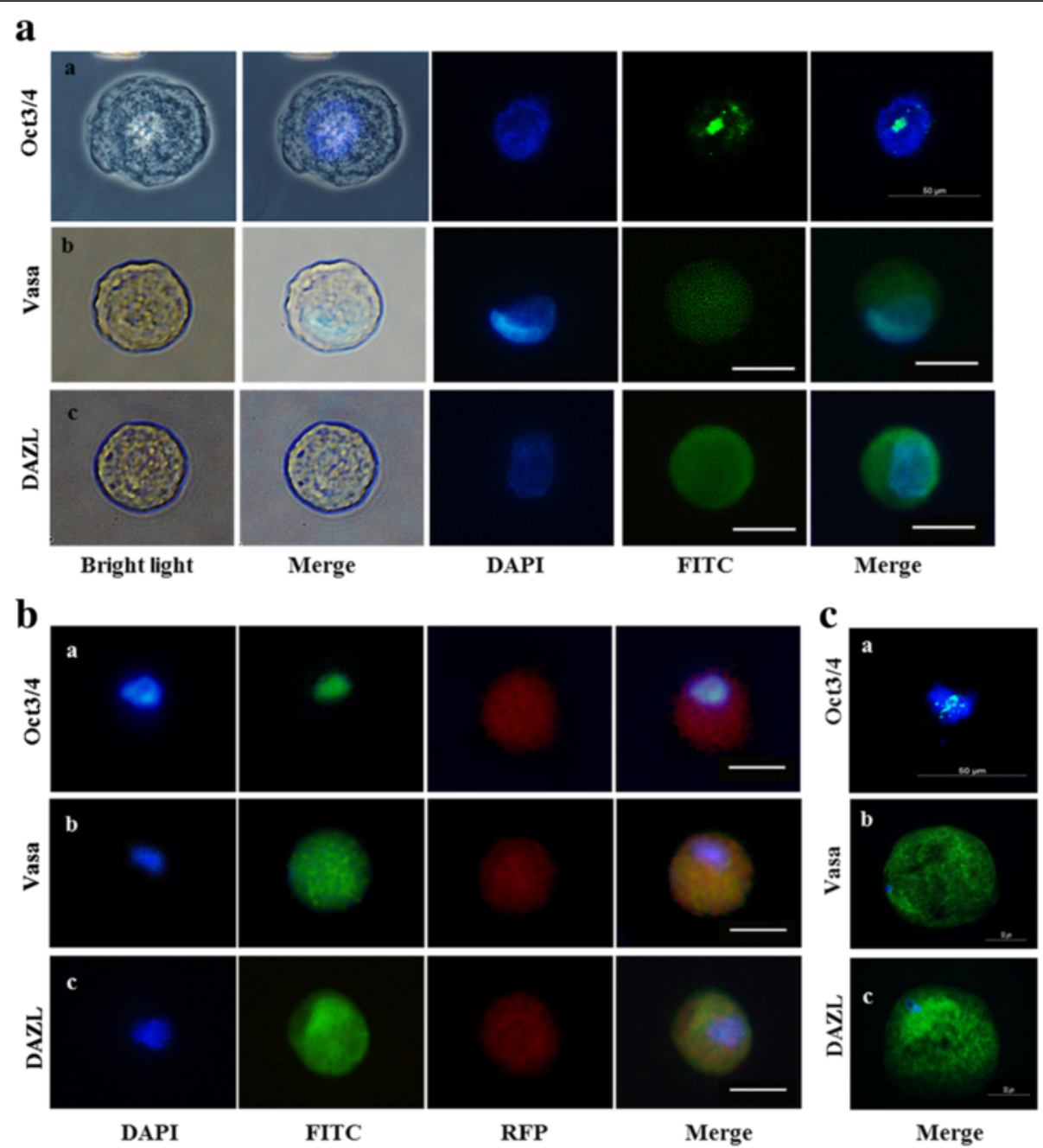

Fig. 5 Characterization of OLCs derived from OvSCs and Oct4-OvSCs by immunocytochemistry. $\mathbf{a}$ and $\mathbf{b}$ Expression of Oct3/4, VASA and DAZL were detected in OLCs from OvSCs and Oct4-OvSCs, respectively. Green fluorescence (FITC) indicates positive reaction and blue fluorescence indicates nuclei (DAPI). Red fluorescence (RFP) indicates transfection of exogenous Oct4. c Expression of Oct3/4, VASA and DAZL in natural oocytes as a positive control. Scale bar $=50 \mu \mathrm{m}$

in this study were more similar to OGSCs in terms of their size and pluripotent status when compare to VSELs. In the present study, we isolated OvSCs from the ovarian cortex and were characterized. Additionally, exogenous Oct4 was transfected to OvSCs to further understand their characteristics and function towards differentiation into OLCs. Both OvSCs and Oct4-OvSCs grew into homogeneous populations with a typical fibroblast-like morphology similar to those of porcine MSCs or MSClike cells derived from various sources including bone marrow, skin, adipose tissue, umbilical cord matrix and ovarian stroma [9, 10, 24-26]. Oct4-OvSCs displayed more population of AP positive cells compared to nonOct4 transfected OvSCs, these observations indicated the enhanced stemness in OvSCs upon transfection with exogenous Oct4 $[12,27]$.
The expression of mesenchymal cell surface markers analyzed using flow cytometer revealed that both OvSCs and Oct4-OvSCs highly expressed CD29, 44 and 90, but negative for hematopoietic cell lineage marker, CD45. The CD marker expression patterns of OvSCs and Oct4-OvSCs were similar with those of porcine MSCs $[9,10,26]$.

The capacity of cell proliferation was promoted by Oct4 transfection in human MSCs [28]. In our study, transfection of Oct4 into OvSCs increased the cell population with S phase of the cell cycle, and Oct4-OvSCs had higher proliferation ability than OvSCs. These results are in agreement with previous findings, wherein Oct4 transfected MSCs resulted in higher proliferation ability $[28,29]$. Oct4, Sox 2 and Nanog are important transcription factors for regulation of pluripotency and maintenance of self-renewal ability in stem cells [21, 30-32]. 


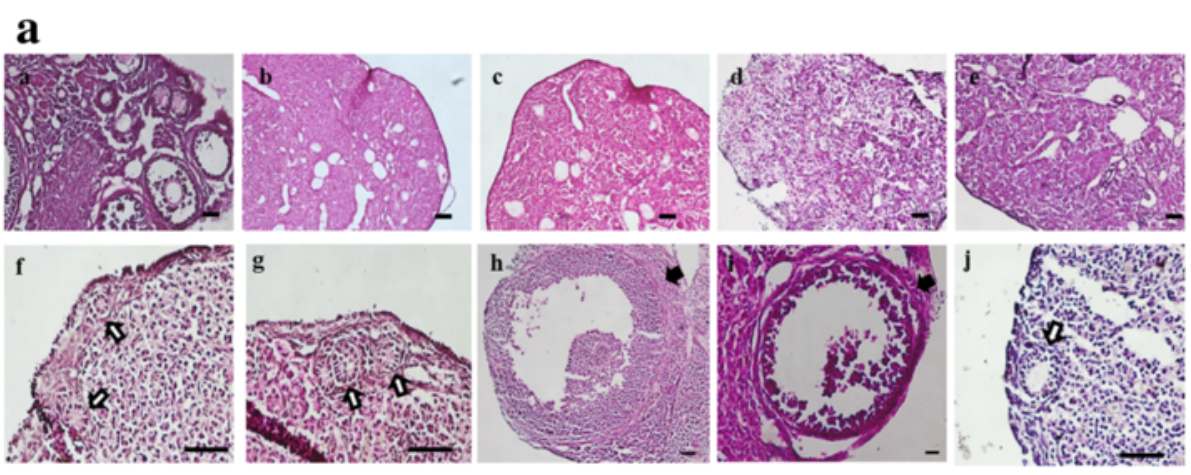

b
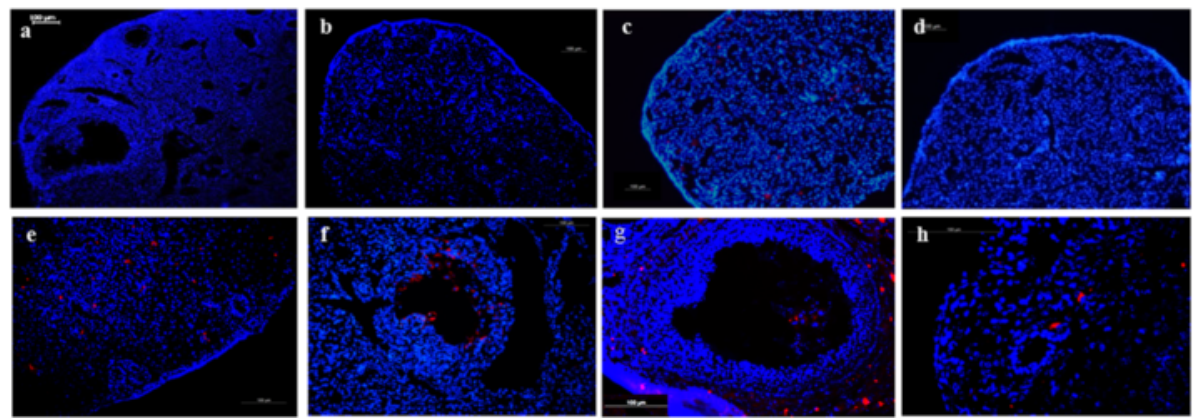

Fig. 6 Histological observation of mouse ovaries following cell transplantation into infertile mouse. a Histological observation by H\&E staining. Image of normal folliculogenesis in BALB/C nude mice ovary (a $a$ ). After twice busulphan injection at 2 weeks interval, follicles and germ cells were destroyed completely in ovaries when observed at 2 and 6 weeks after the second injection ( $\mathbf{a} b$ and $\mathbf{a c}$, respectively). Cells or PBS injection was performed at 2 weeks after the second busulphan injection. Injection of AFs or PBS into sterile ovaries (ad and $\mathbf{a}$, respectively). Transplantation of OvSCs (af and ag) and Oct4-OvSCs (ah-a)). White arrows indicate new primary follicles while black arrows indicate a developing follicle containing theca and groanulosa cells. $\mathbf{b}$ Histological observation by DAPI staining. Image of normal folliculogenesis $(\mathbf{b} a)$. Sterile mouse ovary at 6 weeks after the second busulphan injection ( $\mathbf{b} b$ ). Ovaries injected AFs or PBS (bc and $\mathbf{b} d$, respectively). Transplantation of OvSCs (be) and Oct4-OvSCs (bf-bh). Red fluorescence indicates PKH26 labeled cells and blue florescence indicates nucleus. Scale bar $=100 \mu \mathrm{m}$
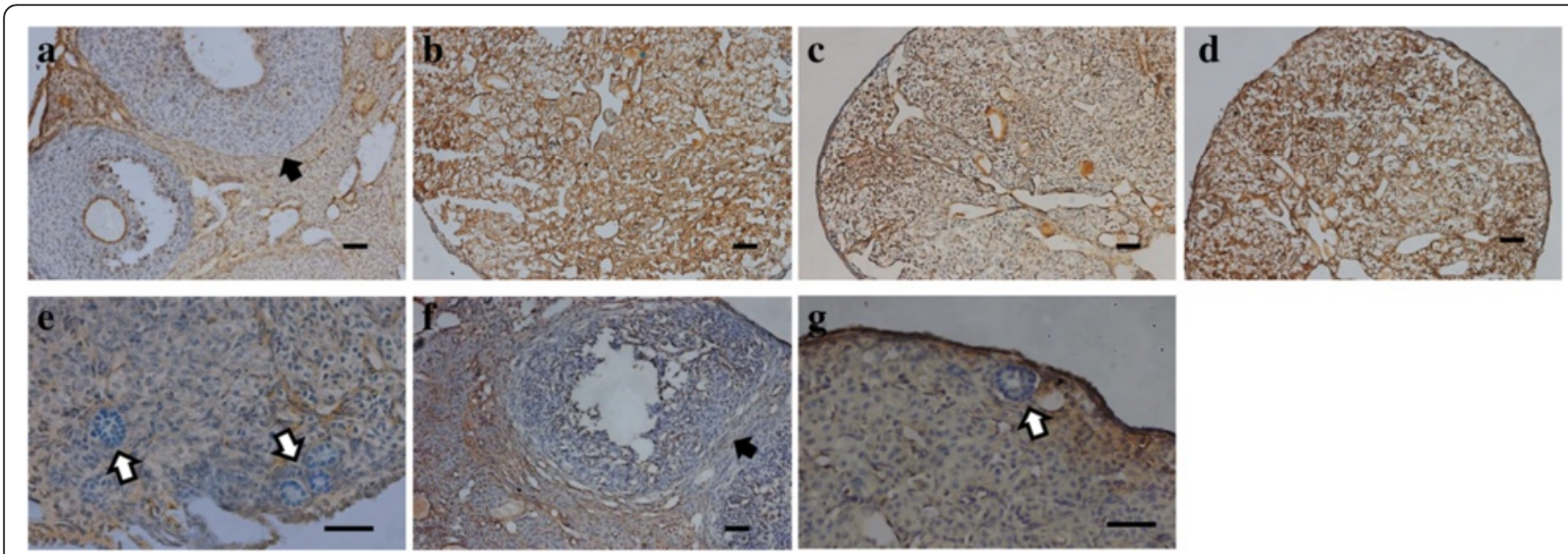

Fig. 7 Expression of estrogen receptor alpha (ERa) in mouse ovaries. Brown color indicates the positive expression of ERa. a Image of normal mouse ovaries. b Sterile mouse ovary at 6 weeks after the second busulphan injection. c and $\mathbf{d}$ AFs or PBS injected mouse ovaries. e OvSCs transplanted ovaries. $\mathbf{f}$ and $\mathbf{g}$ Oct4-OvSCs transplanted ovaries. White arrow indicates the primary follicle which was not expressed ERa. Black arrow means ERa expressed in theca cells. Scale bar $=100 \mu \mathrm{m}$ 

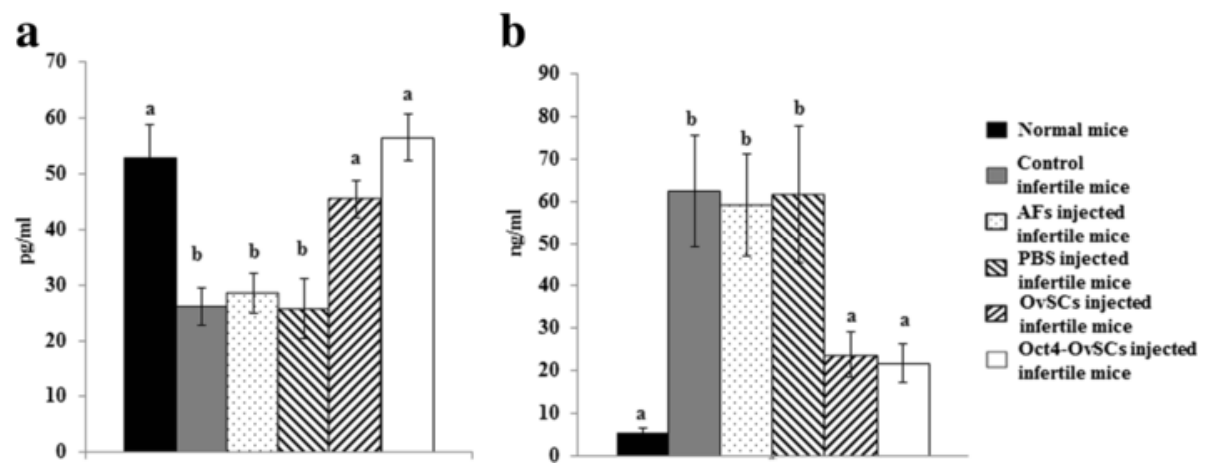

Fig. 8 Hormone (Estrogen and FSH) concentration in mouse serum. a and $\mathbf{b}$ Mouse serum was collected and the concentration of estradiol and FSH were measured by ELISA kit. Different superscript letters represent a significant difference among the groups. Three independent experiments performed in five replicates were used and multiple comparisons were performed by Tukeys test (Mean \pm S.E, $p<0.05$ )

Recent studies have shown the expression of these transcription factors in adult stem cells of various sources, such as skin, adipose tissue, bone marrow and umbilical cord matrix in porcine $[9,12,33]$. Additionally, Song et al. [9] previously reported that these factors were expressed in OvSCs in newborn miniature pig. We detected the expression of Oct4 in OvSCs at mRNA level with no expression at protein level, suggesting these cells may not have the ability to translate to Oct $3 / 4$ protein even though Oct4 mRNA was detected in the cell population. Endogenous Oct4 expression was significantly higher in Oct4-OvSCs at mRNA levels than control OvSCs, and Oct3/4 protein was also detected in Oct4-OvSCs, suggesting that the exogenous Oct 4 transfection has induced an increase in endogenous Oct 4 at mRNA level and might potentiated the cell's ability to translate it into Oct4 protein. The previous reports have demonstrated that Oct4 overexpression had the ability to generate induced pluripotent stem cells in mouse and human [28, 34, 35]. The expression levels of pluripotent genes, including Sox 2 and Nanog, increased by induction to pluripotent stem cell $[28,35]$. Another report [29] has demonstrated that the overexpression of Oct4 in human MSCs derived from the bone marrow at later passage has increased the expression of Nanog. However, the present study showed that the expression levels of Sox 2 and Nanog did not increased by Oct4 transfection in OvSCs, even though transfected cells were expressed mesenchymal lineage markers. This contrasting result might be due to the difference in cell source between the experiments.

In vitro differentiation of OvSCs and Oct4-OvSCs into OLCs [5], after 45 days of differentiation, cells changed their morphology to 'big round cells', which had approximately $50 \mu \mathrm{m}$ diameter and cells revealed zona pellucida-like structure with a nucleus. These findings are in agreement with the earlier reports of OLCs induced from various tissue origins [2, 5, 6, 8, 13]. After induction for 45 days, the number of OLCs, less than
$50 \mu \mathrm{m}$ in diameter, did not differ between Oct4-OvSCs and OvSCs, However, OLCs, more than $50 \mu \mathrm{m}$ in diameter, were more abundant in Oct4-OvSCs when compared to OvSCs. These findings were in accordance with the results of gene expression, in which germ cell specific genes were expressed higher in OLCs from Oct4-OvSCs than those from OvSCs.

To confirm the ability to form OLCs, the expression of transcription factors (Oct4), germ cell and meiosis specific markers (Vasa, GDF9b, C-mos, C-kit, Stella and ZPC), and marker of folliculogenesis (FSHR) was evaluated by RT-PCR and quantified their relative expression using respective band intensities. Oct4, C-kit and ZPC expression was significantly higher in Oct4-OvSCs than OvSCs on day 45 of differentiation, which indicates that Oct4-overexpression resulted in an increase of these gene expressions in differentiated OLCs. The expression of Vasa, GDF9b, C-mos, FSHR, Stella and ZPC was significantly increased after differentiation into OLCs in OvSC or Oct4-OvSCs. Development of OLCs has already been confirmed under different culture conditions with the expression of several oocyte specific genes such as Vasa, DAZL, GDF9b, MLH1, SCP1, PUM1, PUM2, and POU5F1 [36]. Late stage germ cell markers, such as Vasa and GDF9b, and granulosa cell marker, FSHR, were detected at D0 of differentiation in this study, suggesting a possible germ cell and/or granulosa cell contamination in the isolated OvSCs. However, we exclude this possibility, since $40 \mu \mathrm{m}$ cell strainer was used to prevent contamination from primary oocytes when OvSCs were isolated. Further, only cortex part was collected under a microscope to avoid the contamination of granulosa and theca cells. Nevertheless, granulosa cells usually need different culture conditions $[37,38]$ compared to OvSC culture. Therefore, we assume that the granulosa cells should have eliminated during passaging as we used passage 4 cells in our experiment. It has been also reported that ovarian stem cells derived from 
the ovarian surface epithelium (OSE) expresses GDF-9 and VASA in mouse [39], suggesting ovarian stem cells used in the present study could express germ cell marker before differentiating into OLCs. Previous report also demonstrated the similar results for differentiation of ovarian theca stem/stromal cells into OLCs [40]. To further evaluate the expression of Oct3/4, Vasa and DAZL at protein level, OLCs were analyzed by immunocytochemistry. Oct3/4 was strongly expressed in the nucleus of OLCs, whereas Vasa and DAZL were detected in the cytoplasm. These expression patterns were similar to in vitro matured oocytes and suggested that these OLCs have similar protein expression of germ cell markers to natural oocytes.

In vivo folliculogenesis was observed upon transplantation of OvSCs and Oct4-OvSCs into the infertile mice ovaries. The present study showed that busulphan treatment into BALB/C nude mice completely destroyed the follicles and germ cells in ovaries when observed at 2 and 6 weeks after the second injection [3, 17]. Female mouse germline stem cells were transplanted into infertile mouse ovaries and subsequently produced new oocyte and follicle in vivo $[1,3]$. After transplantation of putative theca stem cells into mouse ovary, the cells were observed at the thecal layer of follicles [41]. In the present study, OvSCs and Oct4-OvSCs transplantation into ovaries were clearly contributed to the formation of follicles and its associated cells, such as theca and granulosa cells. OvSCs transplanted into infertile mice resulted in the generation of primary follicles. On the other hand, the ovaries transplanted with Oct4-OvSCs not only generated primary follicles, but also the developing follicles containing theca and granulosa cells. This suggested that Oct4-OvSCs transplantation had greater capacity for folliculogenesis in infertile mice. Further, the developing follicles contained Oct4-OvSCs labeled with PKH26, but primary follicles lacked, suggesting inclusion of Oct4-OvSCs in primary follicles may stimulate the advancement in folliculogenesis. It has been previously reported that granulosa cell nests determine the formation of primordial follicles, as it migrates to the cortex from ovarian stromal layer [42].

Estrogen receptor alpha (ER $\alpha)$ has been shown to be expressed in theca cells and interstitial cells, but not in granulosa cells [43-45]. In the present study, granulosa cells in primary follicles did not expressed ER $\alpha$ in OvSCs or Oct4-OvSCs transplanted ovaries. Further, ER $\alpha$ was expressed in theca cells and interstitial cells in Oct4OvSCs transplanted ovaries. It was demonstrated that ER $\alpha$ expression in OvSCs or Oct4-OvSCs transplanted ovaries were similar to normal mice ovaries.

Infertile mice generated by busulphan injection have been reported to show lower estradiol and higher FSH concentration in serum than the normal mice [46-48]. OvSCs or Oct4-OvSCs transplantation into infertile mice resulted in similar estradiol and FSH concentration as observed in the normal intact mice. Further, Oct4 overexpression in OvSCs resulted in increased level of estradiol concentration when compare to OvSCs upon transplantation into mice. Recent study showed that administration of ovarian cell-like cells differentiated from postnatal mouse skin cells into ovariectomized mice lead to increase in estradiol level. Although these findings are not exactly similar to our observations, these ovarian cell-like cells have function in vivo for recovery of endocrine disorders [49].

\section{Conclusions}

These findings demonstrated that putative stem cells were present in ovarian cortex and have the differentiation ability into OLCs and folliculogenesis in vivo. Oct4overexpression enhanced the AP activity, cell proliferation and differentiation into OLCs compared to OvSCs. In addition, the ability of in vivo folliculogenesis and hormone secretion suggested that Oct4-OvSCs could be considered as a useful in vitro cellular model for understanding the mechanisms of oogenesis and folliculogenesis. However, further studies are required to analyze the over-expression or down-regulation of germ cell specific markers in these stem cells to completely understand the mechanisms of folliculogenesis and oogenesis.

\section{Statement of ethics approval}

This study was approved by the Animal Center for Biomedical Experimentation at Gyeongsang National University via GNU-120404-M0006.

\section{Consent for publication \\ Not Applicable.}

\section{Availability of data and materials \\ Not Applicable.}

\section{Abbreviations \\ GDF9b: growth differentiation factor 9b; OGSCs: ovarian germ stem cells; OvSCs: ovarian stem/stromal cells; VSELs: very small embryonic-like stem cells.}

\section{Competing interests}

The authors declare that they have no competing interests.

\section{Authors' contributions \\ $Y L$ and GR have designed the study and wrote the manuscript. TK contributed to the gene transfection. $Y L, W L, R J$ and $S J$ have participated in the cell culture, differentiation and molecular analysis. YL, TK and $J \mathrm{~L}$ have participated in the in vivo experiments, histological analysis and hormone measurement. SO, SL and $\mathrm{BP}$ have contributed to the analysis of data and discussions. All authors read and approved the final manuscript.}

Funding

This work was supported by a grant from the Bio-industry Technology Development Program (IPET312060-5), Ministry for Food, Agriculture, Forestry and Fisheries, Republic of Korea. 


\section{Author details}

'Department of Theriogenology and Biotechnology, College of Veterinary Medicine, Gyeongsang National University, 501, Jinju-daero, Jinju 660-701, Republic of Korea. ${ }^{2}$ Animal Biotechnology Division, National Institute of Animal Science, Rural Development Administration, Suwon 441-706, Republic of Korea. ${ }^{3}$ Department of Oral and Maxillofacial Surgery, School of Medicine and Institute of Health Science, Gyeongsang National University, Jinju 660-702, Republic of Korea. ${ }^{4}$ Research Institute of Life Sciences, Gyeongsang National University, 501, Jinju-daero, Jinju 660-701, Republic of Korea.

\section{Received: 4 January 2016 Accepted: 31 March 2016} Published online: 12 April 2016

\section{References}

1. Johnson J, Canning J, Kaneko T, Pru JK, Tilly JL. Germline stem cells and follicular renewal in the postnatal mammalian ovary. Nature. 2004;428:145-50.

2. Pacchiarotti J, Maki C, Ramos T, Marh J, Howerton K, Wong J, et al. Differentiation potential of germ line stem cells derived from the postnatal mouse ovary. Differentiation. 2010;79:159-70.

3. Zou K, Yuan Z, Yang Z, Luo H, Sun K, Zhou L, et al. Production of offspring from a germline stem cell line derived from neonatal ovaries. Nat Cell Biol. 2009;11:631-6.

4. Johnson J, Bagley J, Skaznik-Wikiel M, Lee HJ, Adams GB, Niikura Y, et al. Oocyte generation in adult mammalian ovaries by putative germ cells in bone marrow and peripheral blood. Cell. 2005;122:303-15.

5. Dyce PW, Wen L, Li J. In vitro germline potential of stem cells derived from fetal porcine skin. Nat Cell Biol. 2006;8:384-90.

6. Dyce PW, Shen W, Huynh E, Shao H, Villagomez DA, Kidder GM, et al. Analysis of oocyte-like cells differentiated from porcine fetal skin-derived stem cells. Stem Cells Dev. 2011;20:809-19.

7. Linher K, Dyce P, Li J. Primordial germ cell-like cells differentiated in vitro from skin-derived stem cells. PLoS One. 2009;4:e8263.

8. Danner S, Kajahn J, Geismann C, Klink E, Kruse C. Derivation of oocyte-like cells from a clonal pancreatic stem cell line. Mol Hum Reprod. 2007;13:11-20.

9. Song SH, Kumar BM, Kang EJ, Lee YM, Kim TH, Ock SA, et al. Characterization of porcine multipotent stem/stromal cells derived from skin, adipose, and ovarian tissues and their differentiation in vitro into putative oocyte-like cells. Stem Cells Dev. 2011;20:1359-70.

10. Ock SA, Jeon BG, Rho GJ. Comparative characterization of porcine mesenchymal stem cells derived from bone marrow extract and skin tissues. Tissue Eng Part C Methods. 2010;16:1481-91.

11. Sterneckert J, Hoing S, Scholer HR. Concise review: Oct4 and more: the reprogramming expressway. Stem Cells. 2012;30:15-21.

12. Carlin R, Davis D, Weiss M, Schultz B, Troyer D. Expression of early transcription factors Oct-4, Sox-2 and Nanog by porcine umbilical cord (PUC) matrix cells. Reprod Biol Endocrinol. 2006;4:8

13. Cheng $X$, Chen $S, Y u X$, Zheng $P$, Wang H. BMP15 gene is activated during human amniotic fluid stem cell differentiation into oocyte-like cells. DNA Cell Biol. 2012;31:1198-204

14. Yu Z, Ji P, Cao J, Zhu S, Li Y, Zheng L, et al. Dazl promotes germ cell differentiation from embryonic stem cells. J Mol Cell Biol. 2009;1:93-103.

15. Virant-Klun I, Rozman P, Cvjeticanin B, Vrtacnik-Bokal E, Novakovic S, Rulicke $T$, et al. Parthenogenetic embryo-like structures in the human ovarian surface epithelium cell culture in postmenopausal women with no naturally present follicles and oocytes. Stem Cells Dev. 2009;18:137-49.

16. White YA, Woods DC, Takai Y, Ishihara O, Seki H, Tilly JL. Oocyte formation by mitotically active germ cells purified from ovaries of reproductive-age women. Nat Med. 2012;18:413-21.

17. Zhang $Y$, Yang Z, Yang Y, Wang S, Shi L, Xie W, et al. Production of transgenic mice by random recombination of targeted genes in female germline stem cells. J Mol Cell Biol. 2011;3:132-41.

18. Hubner K, Fuhrmann G, Christenson LK, Kehler J, Reinbold R, De La Fuente R, et al. Derivation of oocytes from mouse embryonic stem cells. Science. 2003;300:1251-6.

19. Lv X, Zhu H, Bai Y, Chu Z, Hu Y, Cao H, et al. Reversine promotes porcine muscle derived stem cells (PMDSCs) differentiation into female germ-like cells. J Cell Biochem. 2012;113:3629-42.

20. Medrano JV, Ramathal C, Nguyen HN, Simon C, Reijo Pera RA. Divergent RNA-binding proteins, DAZL and VASA, induce meiotic progression in human germ cells derived in vitro. Stem Cells. 2012;30:441-51.
21. Shi G, Jin Y. Role of Oct4 in maintaining and regaining stem cell pluripotency. Stem Cell Res Ther. 2010;1:39.

22. Takahashi K, Yamanaka S. Induction of pluripotent stem cells from mouse embryonic and adult fibroblast cultures by defined factors. Cell. 2006;126:663-76.

23. Bhartiya D. Ovarian stem cells are always accompanied by very small embryonic-like stem cells in adult mammalian ovary. J Ovarian Res. 2015. doi:10.1186/s13048-015-0200-0.

24. Kang EJ, Lee YH, Kim MJ, Lee YM, Kumar BM, Jeon BG, et al. Transplantation of porcine umbilical cord matrix mesenchymal stem cells in a mouse model of Parkinson's disease. J Tissue Eng Regen Med. 2013;7:169-82.

25. Kumar BM, Maeng GH, Lee YM, Kim TH, Lee JH, Jeon BG, et al. Neurogenic and cardiomyogenic differentiation of mesenchymal stem cells isolated from minipig bone marrow. Res Vet Sci. 2012;93:749-57.

26. Vacanti V, Kong E, Suzuki G, Sato K, Canty JM, Lee T. Phenotypic changes of adult porcine mesenchymal stem cells induced by prolonged passaging in culture. J Cell Physiol. 2005;205:194-201.

27. Kues WA, Petersen B, Mysegades W, Carnwath JW, Niemann H. Isolation of murine and porcine fetal stem cells from somatic tissue. Biol Reprod. $2005 ; 72: 1020-8$

28. Liu TM, Wu YN, Guo XM, Hui JH, Lee EH, Lim B. Effects of ectopic Nanog and Oct4 overexpression on mesenchymal stem cells. Stem Cells Dev. 2009;18:1013-22.

29. Tsai CC, Su PF, Huang YF, Yew TL, Hung SC. Oct4 and Nanog directly regulate Dnmt1 to maintain self-renewal and undifferentiated state in mesenchymal stem cells. Mol Cell. 2012;47:169-82.

30. Kellner S, Kikyo N. Transcriptional regulation of the Oct4 gene, a master gene for pluripotency. Histol Histopathol. 2010;25:405-12.

31. Pan G, Thomson JA. Nanog and transcriptional networks in embryonic stem cell pluripotency. Cell Res. 2007;17:42-9.

32. Rizzino A. Sox2 and Oct-3/4: a versatile pair of master regulators that orchestrate the self-renewal and pluripotency of embryonic stem cells. Wiley Interdiscip Rev Syst Biol Med. 2009;1:228-36.

33. Tang $L$, Yin $Y$, Zhou H, Song G, Fan A, Tang B, et al. Proliferative capacity and pluripotent characteristics of porcine adult stem cells derived from adipose tissue and bone marrow. Cell Reprogram. 2012;14:342-52.

34. Kim JB, Greber B, Arauzo-Bravo MJ, Meyer J, Park Kl, Zaehres H, et al. Direct reprogramming of human neural stem cells by OCT4. Nature. 2009;461:649-53.

35. Kim JB, Sebastiano V, Wu G, Arauzo-Bravo MJ, Sasse P, Gentile L, et al. Oct4-induced pluripotency in adult neural stem cells. Cell. 2009;136:411-9.

36. Richards $\mathrm{M}$, Fong $\mathrm{CY}$, Bongso $\mathrm{A}$. Comparative evaluation of different in vitro systems that stimulate germ cell differentiation in human embryonic stem cells. Fertil Steril. 2010;93:986-94.

37. Morbeck DE, Flowers WL, Britt JH. Response of porcine granulosa cells isolated from primary and secondary follicles to FSH, 8-bromo-cAMP and epidermal growth factor in vitro. J Reprod Fertil. 1993:99:577-84.

38. Gutierrez CG, Campbell BK, Webb R. Development of a long-term bovine granulosa cell culture system: induction and maintenance of estradiol production, response to follicle-stimulating hormone, and morphological characteristics. Biol Reprod. 1997:56:608-16.

39. Joo BS, Jung IK, Park MJ, Joo JK, Kim KH, Lee KS. Differential expression of pluripotent and germ cell markers in ovarian surface epithelium according to age in female mice. Reprod Biol Endocrinol. 2014. doi:10.1186/1477-7827-12-113.

40. Lee YM, Kumar BM, Lee JH, Lee WJ, Kim TH, Lee SL, et al. Characterisation and differentiation of porcine ovarian theca-derived multipotent stem cells. Vet J. 2013:197:761-8.

41. Honda A, Hirose M, Hara K, Matoba S, Inoue K, Miki H, et al. Isolation, characterization, and in vitro and in vivo differentiation of putative thecal stem cells. Proc Natl Acad Sci U S A. 2007;104:12389-94.

42. Bukovsky A, Caudle MR, Svetlikova M, Upadhyaya NB. Origin of germ cells and formation of new primary follicles in adult human ovaries. Reprod Biol Endocrinol. 2004:2:20.

43. Sar M, Welsch F. Differential expression of estrogen receptor-beta and estrogen receptor-alpha in the rat ovary. Endocrinology. 1999;140:963-71.

44. Saunders PT, Millar MR, Williams K, Macpherson S, Harkiss D, Anderson RA et al. Differential expression of estrogen receptor-alpha and -beta and androgen receptor in the ovaries of marmosets and humans. Biol Reprod. 2000;63:1098-105

45. Schomberg DW, Couse JF, Mukherjee A, Lubahn DB, Sar M, Mayo KE, et al. Targeted disruption of the estrogen receptor-alpha gene in female mice: characterization of ovarian responses and phenotype in the adult. Endocrinology. 1999;140:2733-44. 
46. Shirota M, Soda S, Katoh C, Asai S, Sato M, Ohta R, et al. Effects of reduction of the number of primordial follicles on follicular development to achieve puberty in female rats. Reproduction. 2003;125:85-94.

47. Teinturier C, Hartmann O, Valteau-Couanet D, Benhamou E, Bougneres PF. Ovarian function after autologous bone marrow transplantation in childhood: high-dose busulfan is a major cause of ovarian failure. Bone Marrow Transplant. 1998;22:989-94.

48. Yoshida M, Watanabe G, Shirota M, Maekawa A, Taya K. Reduction of primordial follicles caused by maternal treatment with busulfan promotes endometrial adenocarcinoma development in donryu rats. J Reprod Dev. 2005:51:707-14

49. Park BW, Pan B, Toms D, Huynh E, Byun JH, Lee YM, et al. Ovarian-cell-like cells from skin stem cells restored estradiol production and estrus cycling in ovariectomized mice. Stem Cells Dev. 2014;23:1647-58.

\section{Submit your next manuscript to BioMed Central} and we will help you at every step:

- We accept pre-submission inquiries

- Our selector tool helps you to find the most relevant journal

- We provide round the clock customer support

- Convenient online submission

- Thorough peer review

- Inclusion in PubMed and all major indexing services

- Maximum visibility for your research

Submit your manuscript at www.biomedcentral.com/submit 